Journal of Case Reports 2017;7(1):55-57

\title{
Bleomycin Induced Acute Respiratory Distress Syndrome
}

\author{
Amit Daphale $^{1}$, Sourya Acharya ${ }^{1}$, Samarth Shukla ${ }^{2}$, Saish Alegaonkar ${ }^{1}$ \\ Department of ${ }^{1}$ Internal Medicine and ${ }^{2}$ Pathology, Acharya Vinoba Bhave Rural Hospital and Jawaharlal Nehru Medical College, \\ Sawangi (Meghe), Wardha, Maharashtra, India.
}

\author{
Corresponding Author: \\ Dr. Amit Daphale \\ Email: daphaleamit@gmail.com \\ This is an Open Access article distributed \\ under the terms of the Creative Commons \\ Attribution License (creativecommons.org/ \\ licenses/by/3.0). \\ Received : October 2, 2016 \\ Accepted : January 5, 2017 \\ Published : February 10, 2017
}

\begin{abstract}
Background: Bleomycin, glycopeptide antibiotic, is a key chemotherapeutic agent with fatal pulmonary toxicity. Pulmonary adverse events of bleomycin vary from pneumonia to fatal interstitial fibrosis. Case Report: We hereby report a rare case of bleomycin induced acute respiratory distress syndrome in a patient with dysgerminoma ovary. Conclusion: In presence of risk factors, physicians administrating bleomycin should be aware of potential lung toxicity.
\end{abstract}

Keywords: Bleomycin, Dysgerminoma, Pneumonia, Ovarian Neoplasms, Respiratory Distress Syndrome.

\section{Introduction}

With the increasing prevalence of malignancies, use of chemotherapeutic drugs is becoming and more and more common. Inspite of there curative properties, these drugs exhibit some of the fatal side effects. Bleomycin is one of the first drug used in various chemotherapy regimens for various malignancies. Use of this drug leading to various complication has been previously reported. We present such a case of bleomycin induced acute lung injury.

\section{Case Report}

A24 year old female, a known case of dysgerminoma of right ovary on chemotherapy, was admitted in the department of obstetrics and gynaecology for $5^{\text {th }}$ cycle of chemotherapy. She was receiving bleomycin, cisplatin and etoposide. After her $5^{\text {th }}$ chemotherapy cycle, patient developed complaints of breathlessness associated with cough with expectoration. Breathlessness and cough would increase on supine position and patient would feel comparatively better in propped up position. Her oxygen saturation was persistently low between
$80 \%$ (on ambient air) to $85 \%$ (on oxygen at $6 \mathrm{~L} / \mathrm{min}$ ). On examination patient was having tachycardia (pulse rate of 136 beats per minute), tachypnea (respiratory rate: 28/minute) and blood pressure of 100/60 mm Hg. Respiratory examination revealed bilateral reduced air entry with bronchial breathing and coarse crepitations. Rest of the systemic examination was unremarkable.

Patient's complete blood count with differential count, kidney function tests, liver function tests, amylase, lipase levels were unremarkable. Her ultrasonography of abdomen and pelvis failed to reveal anything significant. Chest X-ray showed bilateral consolidation [Fig.1] while HRCT thorax showed diffuse areas of ground glass opacities with patchy areas of consolidation in both lower lobes with air bronchogram suggestive of acute respiratory distress syndrome [Fig.2]. Her $\mathrm{PaO}_{2} / \mathrm{FiO}_{2}$ ratio was less than 200 in view of which patient was shifted to intensive care unit and was intubated.

Patient later succumbed to acute respiratory distress syndrome (ARDS). As no other etiological evidence of ARDS was documented and hence we 
attribute the cause of ARDS to bleomycin induced acute lung injury. Etoposide and cisplatin, the other two drugs of the chemotherapy regime, do not cause pulmonary toxicity as a side effect.

\section{Discussion}

Bleomycin is produced from Streptomyces verticillis $[1,2]$. They are a family of compounds with potent tumor killing properties [1,2]. The skin reactions and pulmonary toxicity are more common with bleomycin due to deficiency of bleomycin hydroloase, while bone marrow suppression is less common [2,3]. Bleomycin is most commonly used as part the standard chemotherapeutic regimen for the treatment of Hodgkin's disease and germ cell tumors [2].

Risk factors associated with increased toxicity to bleomycin include cumulative dose $>300$ units, age $>40$ years, deranged renal function or thoracic radiotherapy $[3,4]$. Bleomycin induced pneumonitis (BIP) has similar clinical presentation to conditions like lower respiratory tract infection, pulmonary metastasis and lymphangitic carcinoma which are commonly seen in patients on chemotherapy [2]. Because of these similarities diagnosis of BIP is often made by exclusion [2]. Our case was one such rare presentation of bleomycin induced pulmonary toxicity. All other possible causes of lung injury in this case were ruled out and finally the lung injury was attributed to bleomycin. Futhermore cisplastin is known to affect the renal functions which further acts as a risk factor for BIP $[1,3,4]$.

Best treatment for BIP is prevention [1-4]. There is lack of good screening tests but regular evaluation by pulmonary function test can be helpful [2-4]. Wherever possible an alternate regimen, if available, should be tried in patients with worsening respiratory function $[2,3,5]$. Glucocorticoids can be helpful till patient is better symptomatically and there is radiological clearance [5].

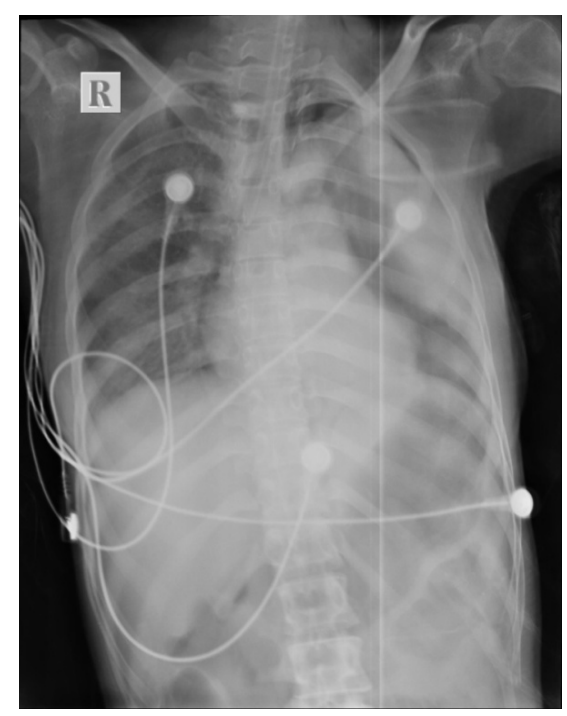

Fig.1: Chest X-ray (PA view) showing bilateral consolidation.

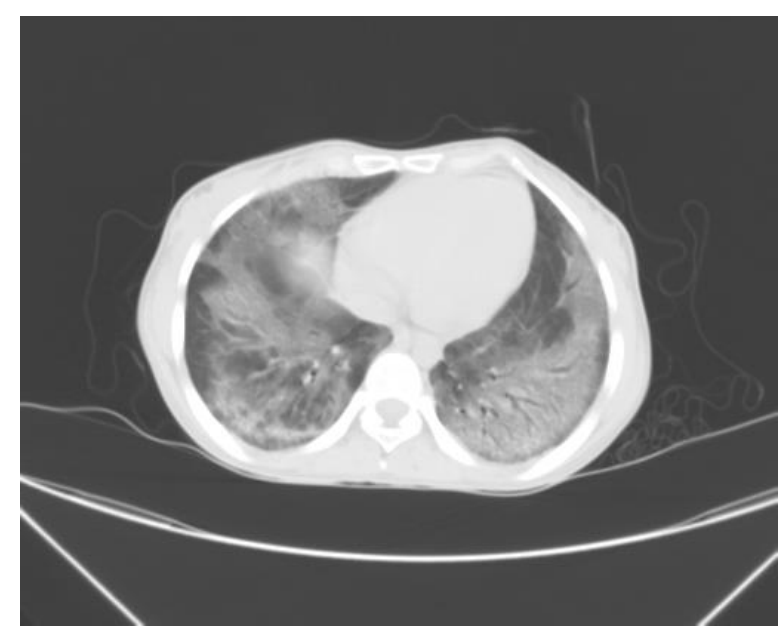

Fig.2: HRCT thorax showing diffuse areas of ground glass opacities with patchy areas of consolidation in both lower lobes with air bronchogram.

\section{Conclusion}

The diagnosis of interstitial lung disease and BIP is challenging in patients with bleomycin. It often depends on clinical, radiological, and cytological findings with exclusion of other etiological factors for lung injury. In presence of risk factors, physicians administrating bleomycin should be aware of potential lung toxicity as we conclude that best treatment for BIP is prevention. 
Contributors: AD contributed to first version of manuscript writing and patient management. SoA, SS and $\mathrm{SaA}$ did the manuscript editing and patient management. All contributors together contributed in reviewing the case literature. $\mathrm{AD}$ will act as guarantor. All authors approved the final version of manuscript. Funding: None; Competing interests: None stated.

\section{References}

1. Hay J, Shahzeidi S, Laurent G. Mechanism of bleomycin induced lung damage. Arch Toxicol. 1991;65:81-94.

2. Reinert T, Clarissa Serodio da Rocha Baldotto, Frederico Arthur Pereira Nunes, Adriana Alves de Souza Scheliga. Bleomycin induced lung injury. Journal of Cancer Research. 2013 (2013);480608.
3. Calcaianu G, Calcaianu M, Gschwend A, Avérous G, Meziani F, Boisramé-Helms J, Kessler R. Fatal Bleomycin-Induced Pulmonary Fibrosis in Case of Seminoma. Open Journal of Clinical and Medical Case Reports. 2016;14:1141.

4. Prakash S, Suri H, Usha G, Gogia A. Bleomycin Induced Pulmonary Toxicity: A Case Report. Internet Journal of Anaesthesiology. 2007;16:1-4.

5. Longo DL et al. Harrisons principles of internal medicine, 2015; $19^{\text {th }}$ edition. Volume 1 . Longo DL et al. Harrison's hematology and oncology. In: Kasper DL, Fauci AS, Longo DL, Hauser SL, Jameson JL, Loscalzo J. Harrisons principles of internal medicine, 2015; $19^{\text {th }}$ edition. Volume 2; New York: McGraw-Hill Education Medical, 2004. 